\title{
EFFEGTS OF SOME GENTRALLY ACTING DRUGS ON FOOD INTAKE OF NORMAL AND HYPOTHALAMUS-LESIONED RATS
}

\author{
YOSHIHIRO MATSUDA \\ Department of Pharmacology, Faculty of Medicine, Kysto University, Sakyo-ku, Kyoto \\ Received for publication April 15, 1966
}

The role of the hypothalamus in the eating act and the regulation of food intake has recently been established in experimental animals by stimulation and destruction of the structure. The hyperphagia with obesity and the aphagia produced by the hypothalamic lesions indicates the localization of two regulatory centers, mutually antagonistic in function, i.e. the satiety center and the feeding center. The satiety center is presumedly involved in regulation of food intake responding to the changes of visceral sensations or blood concentration of metabolic products. The destruction of the ventromedial hypothalamic region has been shown to produce hyperphagia and obesity in a variety of animals.

Apart from the study on the regulation of food intake, attempts have been made to elucidate other aspects of the eating behavior, that is, the motivation mechanism by observing the hunger-motivated behaviors or the conditioned responses. In hypothalamically hyperphagic rats, Miller et al. (1) have shown that their hunger-motivated behaviors are more easily depressed than in normal ones. In the experiments to present the possible involvement of the satiety center in the anorexigenic effect of amphetamine Epstein (2) and Reynolds (3) have found that the reduction of food intake induced by the drug is more marked in hypothalamically hyperphagic rats than in intact ones. From the observation that hypothalamically hyperphagic rats overate the dextrose-added diet, but refused to eat it when cellulose or quinine was added to the food which was still acceptable to normal rats, Teitelbaum (4) regarded them as finicky caters. Epstein (2) suggested that this notion of finickiness might be extended to all feeding situations in the hypothalamus-lesioned hyperphagic rats.

Some pharmacological agents have been known to reduce or enchance the appetite. One of them is amphetamine, and, with other sympathomimetic drugs, it has been often used clinically in obesity control. Schmidt and Van Meter (5) observed a depressing effect of chlorpromazine on food intake in rats, and chlordiazepoxide was reported to augment the food intake and to accelerate the increase of body weight in rats and dogs (6). The clinical observations with reserpine also suggest some effects on the appetite. 
In the present experiment, effects of some centrally acting drugs on the hypothalamic regulatory mechanisms of feeding have been examined by observing the eating behaviors in intact and hypothalamus-lesioned rats.

\section{METHODS}

\section{1) Animals}

Male and female albino rats, weighing 200 to $250 \mathrm{~g}$, were used. They were housed in an individual cage at room temperature of $22 \pm 1^{\circ} \mathrm{C}$, and were fed on the commercial diet, CLEA CA-1, allowing the free access to water. The daily food and water intakes and the body weight were measured individually in the morning throughout the observation period. The observation of these parameters was made for four weeks and in some rats, for two months after the hypothalamic lesion.

\section{2) Lesioning of hypothalamus}

The rats were anesthetized with intraperitoneal injection of $40 \mathrm{mg} / \mathrm{kg}$ of pentobarbital sodium, and their heads were fixed on the stereotaxic instrument of Todai Noken type. After the incision of the scalp, the skull was trephined bilaterally at the site, A. 5.8 to 6.0 and L. 0.7 of de Groot's coordinates, and a monopolar coagulation electrode, made of a stainless steel tube of $0.5 \mathrm{~mm}$ in diameter and insulated except of its tip, was inserted into the ventromedial hypothalamus. The indifferent electrode, a copper plate, was placed on the incised site of the back. An electronic current of 8 to 10 volts was applied, the polarity being changed alternately four times, for 10 to 12.5 seconds. The incised wounds were then sutured and the animal was allowed to recover in the living cage. All the surgical procedures were performed aseptically.

Fifty male and ten female rats were subjected to the operation. Among them 29 males and 5 females exhibited the typical hypcrphagic reaction, and remaining 11 males and 5 females did not show the hyperphagia. Fifteen male and five female rats were kept for 1 to 2 months after the operation allowing free access to food and water, and their behavioral changes including eating and drinking patterns were observed. At the end of this period they were sacrificed for the histological examination of parenchymatous organs. Fourteen hyperphagic males of the operated rats were used for the drug experiment.

\section{3) Drug administration}

Some operated hyperphagic and non-hyperphagic rats as well as intact rats were trained to eat food only for successive 5 hours a day (from 1:00 p.m. to 6:00 p.m.). This eating schedule was started between the 7 th to 10th day after the operation when the animals fully recovered, and in the following 10 days the rats became to show a steady body weight gain or a plateau formation of food intake. The drugs used were methamphetamine hydrochloride, chlorpromazine hydrochloride and chlordiazepoxide hydrochloride. The solutions of the drugs, dissolved in physiological saline in such a concentration that $1 \mathrm{ml}$ contained the respective dose $/ \mathrm{kg}$ were injected intraperitoneally 30 
minutes before the beginning of the daily eating schedule on the test day. The control rats were administered saline alone. The amounts of food intake during the first 2 or 3 hours and also in total 5 hours were estimated. In order to minimize the involvement of nutritional disturbance and persisting drug effects, the administration was made at the interval of at least 2 days. As a rule, two doses of the respective drugs were tested twice in every rat.

\section{RESULTS}

\section{Behavioral changes of the rats with ventromedial hypothalamic lesion}

1) Changes in food and water intake and body weight

About two-thirds of the operated rats exhibited a hyperphagic response. The hyperphagia manifested itself on the first postoperative day in half of the responded animals. One of them showed a voracious eating immediately after awaking from the anesthesia. The hyperphagic response of the other animals was preceeded by some reduction in food intake for one to two days, and thereafter a gradual increase in daily food intake took place in 2 to 3 days attaining a peak value about twice of the preoperative level within a week after the operation. In some of the animals the increased food intake maintained for 1 to 2 months, and in others the food intake gradually decreased but was still larger than the preoperative level. Fig. 1 illustrates a typical change of food and water intake after the operation.

The eating act of the hyperphagic rats was characterized by its persistency and ardency of the muscular movements. The hyperphagic response was often superimposed with the polydipsia and other emotional or motoric behavioral changes, described below, and the rats showed a bizarre behavioral pattern.

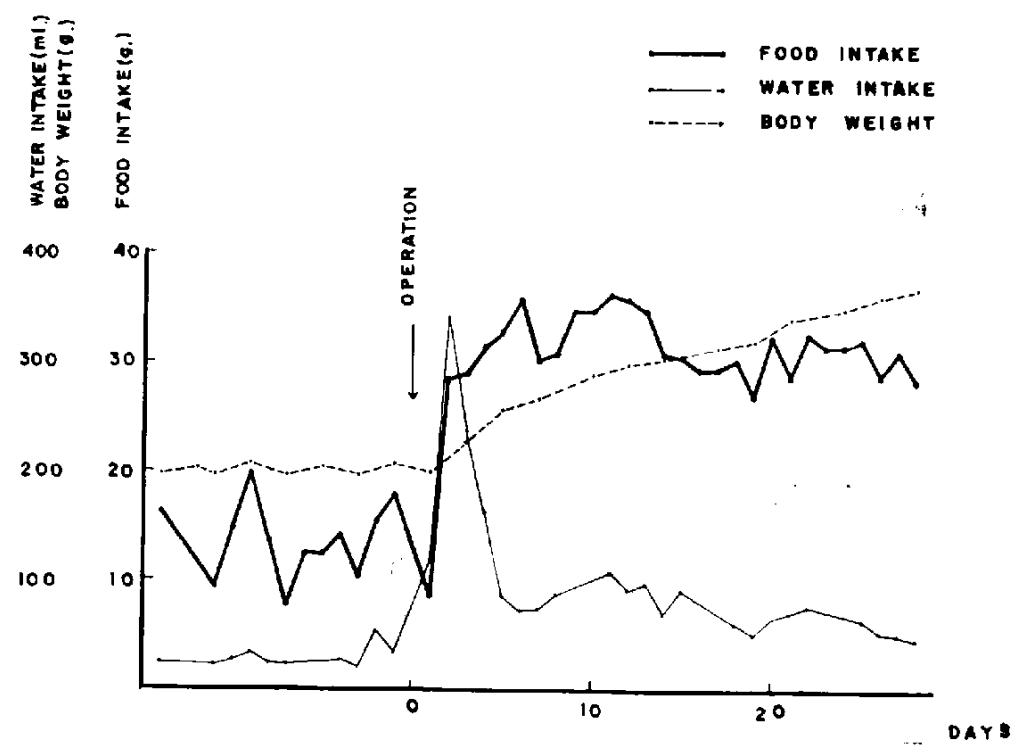

Fig. 1. A typical pattern of changes of food intake and body weight following ventromedial hypothalamic lesion. 
Almost all of the hyperphagic rats showed an increased water intake. A marked but transient increase in water intake attaining to 4 to 8 times the preoperative level occurred in 2 to 3 days after the operation, and thereafter various degrees of polydipsia persisted throughout the observation period. The polydipsia manifested itself more constantly and earlier than the hyperphagia, and, even during a transient postoperative depression of food intake, some rats drank a large amount of water. The polydipsia was associated with an augmented urine flow. Except during early postoperative period, the hyperphagia usually paralleled in its course with the polydipsia. In some rats the polydipsia was more marked than that of hyperphagia, as shown in Fig. 2, and in these rats the hyperphagic response varied daily. Few of the rats whose hyperphagic response was less marked lacked a polydipsic reaction.

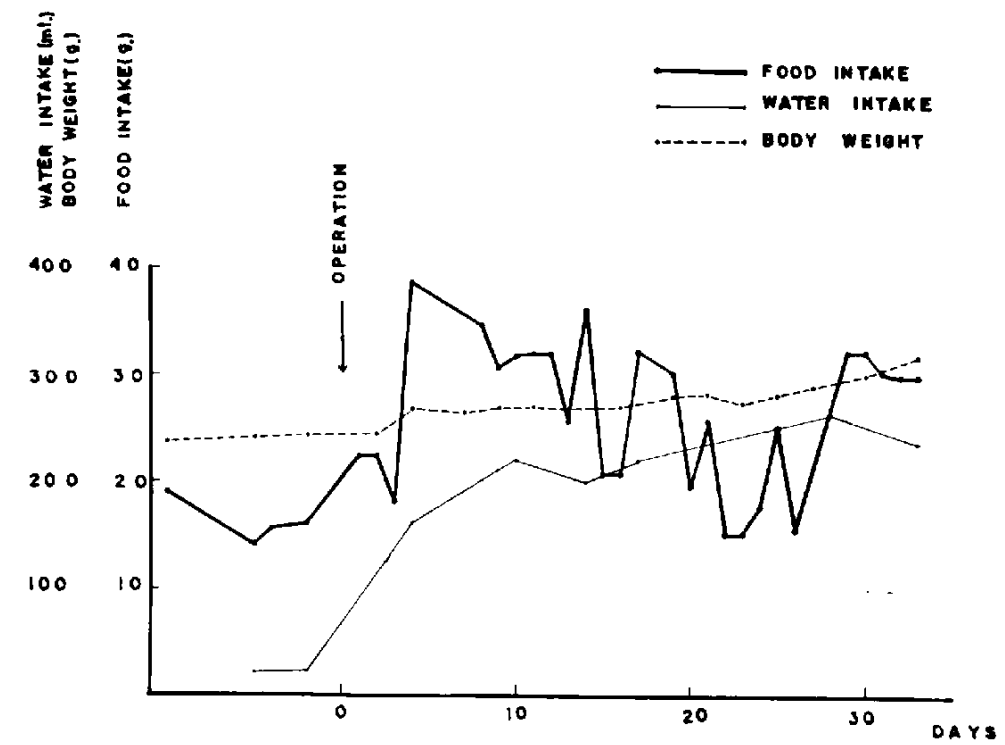

FIG. 2. An anomalous pattern of changes of food and water intake following hypothalamic lesion.

The hyperphagic animals showed an increased rate of body weight gain and became very obese. The mean per cent increase of body weight in 4 weeks after the operation was about $50 \%$, and the maximal increase was $130 \%$.

2) Other behavioral changes

About half of the hyperphagic rats showed a rage reaction. Though they were relatively tame before the operation, they became to show an aggressive behavior such as biting at or attacking against the fingers of operator or other subjects inserted into their cage after the appearance of the hyperphagia. However, this reaction subsided somewhat gradually along with elapse of time.

Four of the hyperphagic rats became hypersensitive to tactile stimuli on the skin. When the back of such rats was touched gently, they showed a prompt avoidance movement with squeaking, and when blowed, they took a fearful attitude such as violent jumping and squeaking. This hypersensitivity to external stimuli was more marked in 
TARLE 1. Behavioral changes and pathological findings in hyperphagic rats.

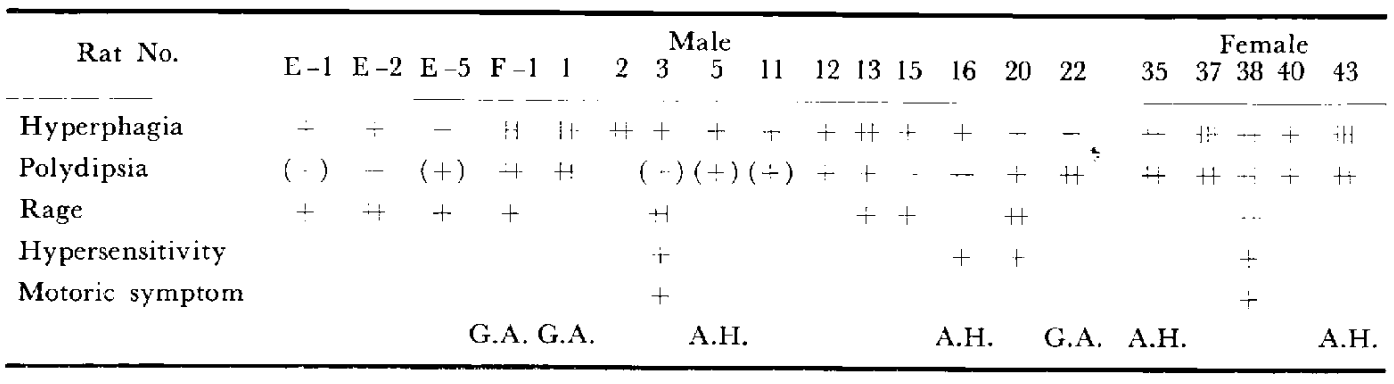

A.H. - Adrenal hypertrophy, G.A.= Gonadal atrophy

the early period after the operation, and gradually subsided thereafter.

In two hyperphagic rats a peculiar motor symptom appeared. In response to extcrnal stimuli they showed a gross tremulous movement of the head, and attacking stimulation could easily make them taking a supine position. 'They tended to rotate around on walking, and their eating movement became awkward. These anomalous movements were never improved throughout the observation period. Table 1 summarizes the above mentioned behavioral changes in the hyperphagic rats. Sixteen of 60 operated rats did not cxhibit the hyperphagia. Usually their food intake was transiently depressed in the early postoperative days, but returned to the preoperative level before long. All of them showed no behavioral changes and was indistinguishable from the unoperated rats except the two, one of which cxhibited the hypersensitive reaction with the anomalous movement mentioned above, and the other manifested the polydipsia with the motor symptom.

\section{Effect of cen'rilly acting drugs}

Four to 6 days were required until the intact rat learned to take the previous diurnal amount of food according to the daily 5 hours of the eating schedule. On the other hand, the hypothalamus-lesioned rats needed two or three more days for attaining a constant level of food intake during 5 hour period, and moreover, the mean value of food intake at the time of plateau formation was slightly less in the operated rats than in the intact ones. One of the operated but non-hyperphagic rats, whose daily intake on the eating schedulc never exceeded $10 \mathrm{~g}$, about a half of the value in the intact rats, was cxcluded from the experiment. Drug effects were observed in the trained intact, the hyperphagic and the operated non-hyperphagic rats.

\section{1) Methamphetamine}

Fig. 3 shows the mean values of food intake by the intact, operated non-hyperphagic and hyperphagic rats in the first 2 and total 5 hours of the eating schedulc under the treatment of methamphetamine. Without methamphetamine the food intake during 5 hours did not differ significantly betwcen the respective groups of rats. Methamphetamine in dose of $2 \mathrm{mg} / \mathrm{kg}$ reduced the food intake in all groups and the largest reduction was observed in the hyperphagic rats. In the dose of $3 \mathrm{mg} / \mathrm{kg}$ the differences betwcen three groups became apparent, i.e. being the hyperphagic rats most sensitive and the 


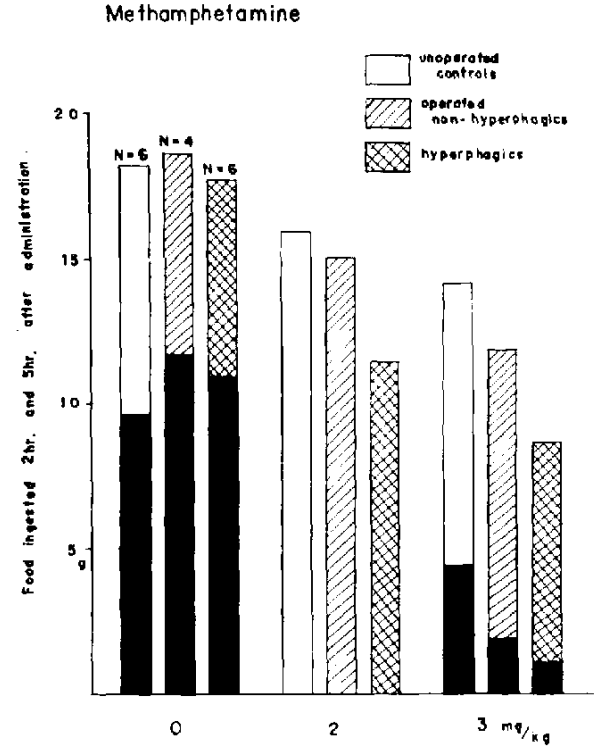

FIG. 3. Amounts (g) of food eaten in a daily 5 hours of cating schcdule by normal, operated non-hyperphagic and hypesphagic rats under the treatment of methamphetamine.

Black columns indicate food intake in first 2 hours.

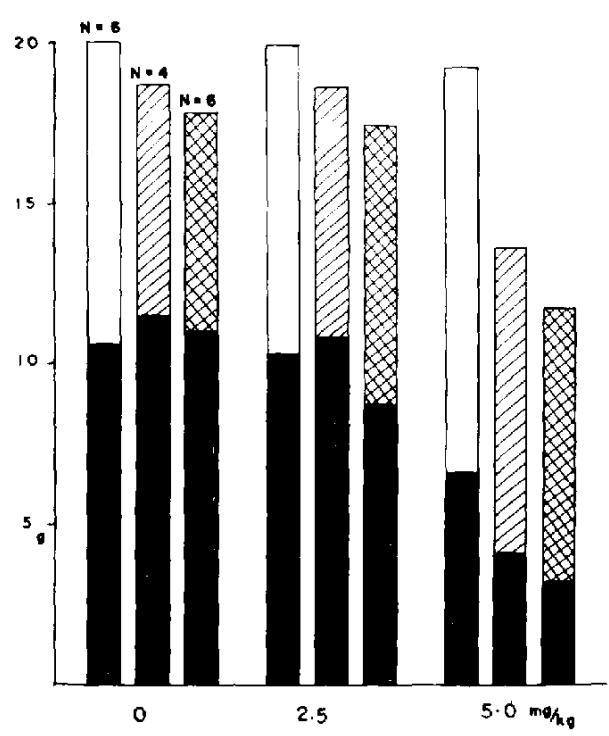

FiG. 4. Amounts (g) of food eaten in a daily 5 hours of eating schedule by normal, operated non-hyperphagic and hyperphagic rats under the treatment of chlorpromazine.

Black columns indicate food intake in first 2 hours.

normal un-operated rats least sensitive. A similar order of the sensitivity in food intake was also observed during the first 2 hours of eating schedule. The mean reductions of the intake during 5 hours in the intact, the operated non-hyperphagic and the hyperphagic rats received $3 \mathrm{mg} / \mathrm{kg}$ were 22,37 and $51 \%$, and those during the first 2 hours were 53,84 and $90 \%$ of the level in the untreated rats, respectively.

A significant behavioral excitements such as locomotion, seeking and running movements was induced by the dose of $3 \mathrm{mg} / \mathrm{kg}$ of methamphetamine. However, no qualitative and quantitative difference in behavioral excitements was observed between the three groups. The operated rats were often quite indifferent to diet during the behavioral excitement caused by methamphetamine, while the eating behavior of the intact rats was not completely suppressed. When the methamphetamine excitement subsidcd 1 to 1.5 hours after the administration, the intact rats restored their usual eating activity, but there remained still some suppression of cating act in the operated group. Thus the depressing effect of methamphetamine on eating was more marked during the behavioral excitement, and more lasting in the operated rats than in the normal un-operated controls.

\section{2) Chlorpromazine}

The effects of 2.5 and $5 \mathrm{mg} / \mathrm{kg}$ of chlorpromazine on food intake during the first 2 and total 5 hours of the eating schedule are shown in Fig. 4 . The dose of $2.5 \mathrm{mg} / \mathrm{kg}$ 
produced no significant effect on food intake during the 5 hours, but the hyperphagic rats were slightly reduced in their intake during the first 2 hours. Although the dose of $5 \mathrm{mg} / \mathrm{kg}$ did not affect the food intake during 5 hours in the intact rats, it depressed significantly the intake in the operated rats. During the first 2 hours, the dose of $5 \mathrm{mg} /$ $\mathrm{kg}$ of the drug reduced the food intake in all threc groups of rats, and the mean reduction in the intact, the opcrated non-hyperphagic and the hyperphagic rats were 38,65 and $70 \%$ of the level in the untreated rats, respectively. The depression of food intake during the early period of eating schedule in the un-operated rats was almost completely compensated during the later period.

The dose of $2.5 \mathrm{mg} / \mathrm{kg}$ did not affect the spontaneous activity in any groups of rats, while that of $5 \mathrm{mg} / \mathrm{kg}$ produced a significant sedation. However, no difference in sedation was detected between the three groups. The sedated animals were not indifferent to the diet, but on the whole, their eating act was slower in movement and shorter in duration.

\section{3) Chlordiazepoxide}

The effects of 5 and $10 \mathrm{mg} / \mathrm{kg}$ of chlordiazepoxide on the food intake in the intact and the hyperphagic rats are presented in Fig. 5. Due to incomplete training of the hyperphagic rats for the scheduled eating, the intake of the hyperphagic rats was relatively less than that of the un-operated controls. Both doses of chlordiazepoxide increased slightly the food intake during 3 and 5 hours in both groups of rats, but no significant difference coulud be seen in rates of increase between both groups. The mean increase of the food intake during 5 hours was $8 \%$ in the intact rats and $12 \%$ in the hyperphagic rats. No depression on the eating activity in the rats received 10 $\mathrm{mg} / \mathrm{kg}$ was observed despite the appearance of a considerable sedation and a slight ataxia of the hind limbs, and this indicated that the motor impairment caused by chlordiazepoxide had little infuence on eating behavior.

\section{Histological findings of hypothalamus-lesion- ed rats}

The autopsy findings of operated hyperphagic rats sacrificed 1 to 2 months after

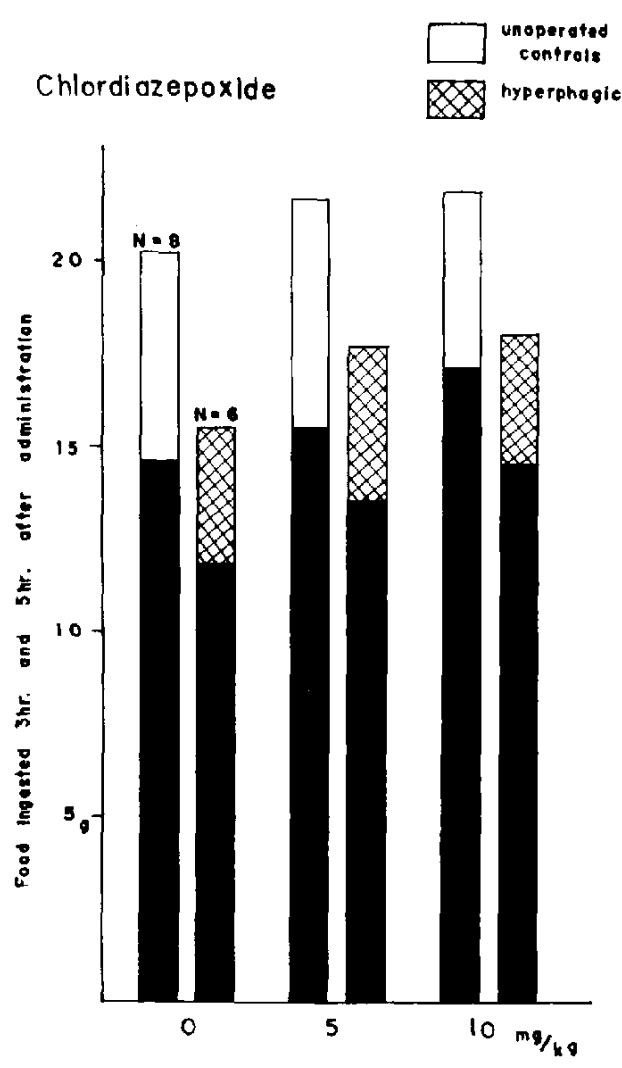

FIG. 5. Amounts (g) of food eaten in a daily 5 hours of eating schedule by normal and hyperphagic rats under the treatment of chlordiazepoxide.

Black columns indicate food intake in first 3 hours. 
the lesioning were as follows. The most characteristic was a marked deposition of fats in the abdominal cavity, especially in the retroperitoneal space. The adrenals were completely burried in fats, which also surrounded the kidneys. The stomach was generally dilated. The liver in many of the hyperphagic rats exhibitcd small white discoloration indicating the increased deposition of fat and sometimes the fatty tissues obliterated the space between the liver and the diaphragm.

In hyperphagic rats the changes in the weight of parenchymotous organs were most marked in the liver. Histologically, the protoplasm of acinal liver cells was diffusely filled with gross and fine vacuoles indicating a fatty deposition. Some of the hyperphagic rats showed an increase in weight of adrenals caused by a cortical hypertrophy, and some showed a marked testicular atrophy associated with a decrease in weight of prostate. The anterior hypophysis sometimes showed a sign of proliferation of basophilic cells. No pathological finding was detected in kidney, spleen, thyroid gland and pancreas in weight and histological finding.

The operated non-hyperphagic rats showed no pathological finding on autopsy and on histological examination except one rat, which showed a slight increase in weight of adrenals.

The brains of the hypothalamus-lesioned rats were fixed with absolute alcohol and embedded in paraffine, then the serial 40 micra cut of hypothalamic region was made. The site and the extent of lesions were examined with hematoxyline-eosine staining. The uni- or bilateral lesions of the ventromedial hypothalamus were confirmed in hyperphagic rats, but the relationship between lesions and the hypothalamic ventromedial nucleus was not definitely verified. The hyperphagia showed a tendency to be proportional to the extent of hypothalamic lesions, but in some animals even a minute lesion induced a conciderable hypcrphagia. The polydipsia was more marked in the rats with lesions located somewhat caudally and ventrally. The rats which showed the rage reaction exhibited a bilateral destruction of the ventromedial hypothalamus. The operated but non-hyperphagic rats showed the lesions dislocated more laterally or caudally from the ventromedial area.

\section{DISGUSSION}

The development of hyperphagia in rats by uni- or bilateral electro-coagulation of the ventromedial hypothalamus was confirmed in the present experiments. Since a minute lesion produced a hyperphagia, it seems that the destruction of a circumscribed area of the structure is suffice to induce the response. Even a unilateral lesion could causc a hyperphagic response, as Mayer and Barnett (7) have reported. The increase in body weight was accelerated in accordance with the development of hyperphagia, and, since the hyperphagia maintained during 1 to 2 months after the operation, the drug effects were observed during the dynamic stadium of the hyperphagic response.

Most of the hyperphagic rats exhibited a polydipsia. The increase in water intake was usually more marked than that in food intake. The polydipsia was always asso- 
ciated with polyuria, which was likely to derive from the diabetes insipidus caused by lesion in the supraoptico- and/or paraventriculo-hypophysial tract. The possibility of separating the polydipsia from the hyperphagia was shown in the rats which exhibited either a hyperphagia or a polydipsia alone.

It is well assumed that the hypothalamic lesions affect the hypophysial regulatory mechanisms on the endocrine function. In this cxperiment, some of the hyperphagic rats showed a hypertrophy of the adrenal cortex or an atrophy of the gonadal organs. However, since both of these changes were not observed concomitantly, the hypothalamic areas responsible for each response are seemingly different. The gonadal atrophy and the abolition of oestrus caused by the destruction of the hypothalamic ventromedial nucleus have been reported (8), while the exact location concerning the regulation of the adrenocortical function remains to be settled. The changes of endocrine function may possibly be involved in the development of obesity. The same possibility was suggested by Poircr et al. (9), who reported that the obesity developed in the hypothalamic hyperphagic monkey in spite of the restricted food. Bach et al. (10) also found marked deposition of fat in the cat with the lesion in the ventromedial nucleus region under the similar condition.

The increased aggressiveness of the hyperphagic rats is likely to be the same kind of response observed by Wheatly (11) in cats lesioned in the hypothalamic ventromedial nucleus. The hypersensitive response to external stimuli observed in some operated rats may be an abortive type of the rage reaction, i.e. a kind of fear sensation. The possibility that fear or extreme irritability manifests as a rage should be taken into consideration.

In order to analyse the effects of the centrally acting drugs on food intake in the intact and hypothalamus-lesioned rats, the animals were trained to eat for 5 hours a day. In this situation the hyperphagic rats did not overeat, as Epstein (2) had found in a daily 6 hour eating schedule. Some of the hyperphagic rats showed a less food intake than the intact rats. This may be a reflection of a decreased ability to acquire the training. The food intake during the early stadium of a daily cating schedule is presumedly motivated by hunger sensation caused by fasting for 19 hours, and, since the animals may eat with their maximal effort until satiation, the amounts of food eaten during the early period do not differ between the controls and the operated rats. However, the food intake during the late period of the eating schedule may be affected by a learning process, by which the food intake is superimposedly activated. Though the training reduced the body weight at first, a later recovery to the previous level and a gradual increase of body weight indicate that the animals adapted to the eating schedulc. The adaptation was attained more efficiently in the intact rats than in the hyperphagic ones.

In accord with the results shown in effects of amphetamine by Epstein (2) and Reynolds (3), the reduction in food intake by $3 \mathrm{mg} / \mathrm{kg}$ of methamphetamine was most marked in the hyperphagic rats. The reduction in the hypothalamus-lesioned but nonhyperphagic rats was intermediary between those in the intact and the hyperphagic 
rats. This evidence indicates that a diffuse hypothalamic lesion results in an increased sensitivity to anorexigenic effect of methamphetamine. Similarly with methamphetamine, $5 \mathrm{mg} / \mathrm{kg}$ of chlorpromazine reduced the food intake most markedly in the hyperphagic and then in the operated non-hyperphagic rats but slightly in the intact ones. Though the exact mechanism of methamphetamine and chlorpromazine to reduce the food intake is not fully known, these drugs have been reported to reduce the water intake as well (12, 13). Andersson and Larsson (13) showed that at least some central actions of methamphetamine werc responsible for the depression of food and water intake, since in the "prefontal lobotomized" $\operatorname{dog}$ the amphetamine effect on water intake disappeared, and the dose of amphetamine 50 to $100 \%$ more than in intact animals was necessary to block the eating in the lobotomized animal. The present experiment shows that the "satiety" center is not at least the primary site of action of methamphetamine and chlorpromazine, though Brobeck et al. (14) found and enhencement of local electrical activity in the hypothalamic ventromedial nucleus following the administration of amphetamine derivatives in cats.

An increased food intake by chlordiazepoxide was firstly described by Randall et al. (6). Though a similar sequence was observed in the present experiment, no significant difference in increase between the intact and the hyperphagic rats was detected. Teitelbaum (4) has regarded the bchavioral responses of hypothalamic hyperphagic rats as an exaggeration of normal bchaviors, but in the present experiment no such exaggeration of response could be detected with chlordiazepoxide. Revicwing the results presented by Miller et al. (1), Epstein (2) and Reynolds (3), the hyperphagic rats are likely to respond in an exaggerated manner to the agents which depress the food intake.

The exaggerated response of the cating behavior to methamphetamine and chlorpromazine is observed in the rats with hypothalamic lesions in the sites other than "satiety" ccnter. Therefore, furthcr study to correlate the exaggerated response with the hypothalamic lesion is necded. In addition, it should be pointed out that some of the hypothalamus-lesioned rats were poor in ability to adapt to the eating schedule. Therefore, the possible internal or external disturbance caused by the hypothalamic lesions may affect the eating adaptability and thus affect the food intake. These possibillity suggests strongly that the hypothalamic lesion causes not only the deficiency of the lesioned structure but also the overall interference with the correlating function.

\section{SUMMARY}

An observation on food and water intake as well as on other behaviors and histological picture of the parenchymatous organs was made following the ventromedial hypothalamic lesions in rats. Effects of some centrally acting drugs on food intake of normal and hypothalamus-lesioned rats were observed under the 5 hours of daily eating schedule.

The rats lesioned uni- or bilaterally in the hypothalamic ventromedial area showed a hyperphagia, which persisted at least for 1 to 2 months after the operation. In many 
of the hyperphagic rats, a polydipsia was observed concomitantly. Other behavioral changes such as an increased aggressiveness, a hypersensitivity to external stimuli and a peculiar motor symptom were noted in some of the operated rats. Adrenal hypertrophy and gonadal atrophy as well as the marked deposition of fat in tissues, especially in liver observed in a few of operated rats were characteristic findings in the endocrine organs.

Methamphetamine in the dose of 2 to $3 \mathrm{mg} / \mathrm{kg}$ reduced food intake in the 5 hours of eating schedule most markedly in hyperphagic rats and then in operated non-hyperphagic ones. The same order of sensitivity was observed with chlorpromazine, which reduced the food intake. Chlordiazepoxide in the dose of 5 to $10 \mathrm{mg} / \mathrm{kg}$ increased slightly the food intake in both normal intact and operated hyperphagic rats, but no significant difference in rate of increase was found between the two grougs.

\section{REFERENCES}

1) Miller, N.E., Bailey, C.J. And Stf.vfnson, J.A.F. : Science 112, 256 (1950)

2) Epstein, A.N. : J. comp. physial. Psychol. 52, 37 (1959)

3) ReYNOLDS, R.W. : lbid, 52, 682 (1959)

4) Teltellaum, P. : Mid. 48, 156 (1955)

5) Schmidt, H.Jr. ANd Van Meter, W.G. : Ibid. 51, 29 (1958)

6) Randall, L.D., Schallek, W., Heisf, G.A., Keinh, F.F. Ayn Bafion, R.E. : J. Pharmacol. 129, $163(1960)$

7) Mayer, J. and Barnet, R.J. : Science 121, 599 (1955)

8) Harris, G.W. : Handbook of Physiology, Sect. I., 2, p. 1037, American Physiological Society, Washington (1960)

9) Poirer, L.J., Mouren-Mathiel, A.M. And Richre, C.L. : Physiologist 4, 88 (1961)

10) Bach, L.M.N., O'Bries, G.P. ANo CoOprer, G.P. : Progress in Brain Research, Vol. 5, Flsevier Publishing Co., Amsterdam (1964)

11) Wheatiey, M.D. : Arch. Neurol. Psychiat., Chicago 52, 296 (1944)

12) Archer, J.D. : Fed. Proc. 13, 332 (1954)

13) Andersson, B. And Larsson, S. : Acta physiol. scand. 36, 22 (1956)

14) Brobeck, J.R., Larsson, S. And Reyes, R. : J. Physiol. 132, 358 (1956) 